\title{
Evidencia en el manejo del dolor de espalda crónico con el método McKenzie
}

\author{
Evidence in the management of chronic back pain with the McKenzie method
}

\author{
B. Rodríguez Romero, A. Martínez Rodríguez, L. Carballo Costa, F.J. Senín Camargo, \\ G. Paseiro Ares e I. Raposo Vidal
}

\begin{abstract}
Resumen
Objetivo: Revisar la evidencia científica publicada sobre el método McKenzie en dolor lumbar.

Estrategia de búsqueda: Búsqueda en la base de datos Medline, en la página web del Instituto McKenzie y análisis de la evidencia publicada por May y Donelson (2008).

Selección de estudios y datos: Se limita la búsqueda a los últimos 5 años. Se realiza una búsqueda inversa y un filtrado manual centrado en extraer resultados estrechamente relacionados con la temática de búsqueda y en función del nivel de evidencia de los artículos.

Síntesis de resultados: Se encuentra evidencia sobre diversos aspectos del método McKenzie: fiabilidad interobservador como método exploratorio, especificidad del fenómeno de centralización y eficacia comparado con otros tratamientos.

Conclusiones: El método McKenzie presenta eficacia similar a los ejercicios de estabilización y una tendencia favorable comparándolo con los ejercicios de fortalecimiento. El fenómeno de centralización tiene valor pronostico positivo. Presenta una alta fiabilidad como método de exploración.
\end{abstract}

\begin{abstract}
Objective: To review the scientific evidence published on the McKenzie method for back pain.

Research strategy: A search was made in the Medline database, and on the McKenzie Institute website, and the evidence published by May and Donelson (2008) was analyzed.

Studies and data selection: The search was limited to the last five years. A reverse search and a manual filtering (as a function of the level of evidence) focused on extracting results closely related to the subject under research were performed.

Results summary: Evidence on the different aspects of the McKenzie method has been found: inter-examiner reliability as exploratory method, specificity of the centralization phenomenon, and effectiveness compared to other treatments.

Conclusions: The McKenzie method has similar effectiveness as that of stabilization exercises with a favorable tendency in comparison with strengthen-building exercises. The centralization phenomenon has a positive prognostic value. The method present high reliability as an exploratory method.
\end{abstract}

Palabras clave

Fisioterapia; Evidencia; Dolor lumbar; Ejercicio; Columna vertebral

Keywords

Physical therapy; Evidence; Low back pain; Exercise; Spine 


\section{Objetivo}

El objetivo de este artículo ha sido revisar la evidencia científica publicada sobre el método McKenzie en el dolor lumbar.

\section{Estrategia de búsqueda}

La búsqueda de información ha constado de los siguientes pasos:

- En primer lugar, se realiza una búsqueda en la base de datos Medline (www.ncbi.nlm.nih.gov/pubmed) con la siguiente estrategia:

("Low Back Pain"'[Mesh] OR "Lumbosacral Plexus"'[Mesh] OR "low back pain'"[ti] OR “chronic pain"'[ti]) AND ("'Physical Therapy Modalities"'[Mesh] OR "Physical Therapy (Specialty)"'[Mesh] OR “"Musculoskeletal Manipulations"'[Mesh] OR “Exercise Movement Techniques" [Mesh] OR "physiotherapy"'[ti] OR "physical therapy"[ti]) AND ("McKenzie"[tiab] OR "centralisation" [tiab]).

Se obtienen 51 resultados.

- En segundo lugar, se analiza la literatura contenida en la página web del Instituto McKenzie, obteniendo 36 resultados.

- En tercer lugar, se analiza la evidencia publicada por May y Donelson (2008).

\section{Selección de estudios y datos}

Se limita la búsqueda a los últimos 5 años y se realiza una búsqueda inversa en busca de nuevos artículos no reportados en la estrategia marcada.

Para finalizar, se realiza un filtrado manual a los resultados obtenidos, obteniendo un resultado final de 48 referencias bibliográficas.

Este filtrado manual se realiza siguiendo dos premisas. La primera se centra principalmente en extraer aquellos resultados relacionados estrechamente con la temática de búsqueda, es decir, con la pregunta por investigar. La segunda está relacionada con la selección de los artículos en función de su nivel de evidencia, seleccionando de esta manera revisiones sistemáticas, guías de práctica clínica basada en evidencias y ensayos clínicos aleatorizados.

Síntesis de resultados: se encuentra evidencia sobre diversos aspectos del método McKenzie que presentamos en los siguientes subapartados: definición, historia y descripción general; propuesta de clasificación diagnostica de pacientes con dolor lumbar; enfoque terapéutico en función de la clasificación, y evidencia sobre la eficacia del método (que a su vez exponemos en los subapartados de guías de práctica clínica, revisiones sistemáticas, estudios específicos sobre el fenómeno de centralización y ensayos clínicos randomizados).

Definición: el método McKenzie es un método integral de abordaje de personas con dolor de espalda que incluye la exploración del paciente, su clasificación en 3 síndromes mecánicos y una propuesta de estrategias terapéuticas para cada síndrome. Aunque ha recibido diferentes nombres, por ejemplo "Programa McKenzie", "Ejercicios de McKenzie", etc., lo más correcto es referirse a éste como Method of Mechanical Diagnosis and Therapy (MDT, 'método de diagnóstico y tratamiento mecánico'). Es importante mencionar que en muchas ocasiones se hace referencia al método McKenzie de una forma errónea, porque en lugar de incluir los 3 componentes del método (exploración, clasificación 'n y tratamiento) sólo se contempla la parte terapéutica. Se alude al método cuando en realidad sólo se maneja una parte de éste de forma aislada, por ejemplo, los ejercicios o posturas de extensión. May ${ }^{1}$ sugiere que es preferible identificar específicamente la intervención y citarla como tal. El buen uso del lenguaje permitirá superar uno de los errores más frecuentes que es confundir o asociar ejercicios de extensión con el método McKenzie, lo que implica una simplificación de éste. Actualmente, el método McKenzie es un método de terapia manual reconocido por la International Federation of Orthopaedic Manipulative Physical Therapists, subgrupo de la World Confederation for Physical Therapy, representante en materia de terapia manual ortopédica, especialidad de la fisioterapia para el tratamiento del dolor y patología de la columna vertebral y extremidades.

Historia y descripción general: uno de los primeros hallazgos que se sitúa como punto de partida para el desarrollo del método McKenzie se remonta al año 1958. La anécdota que McKenzie relata es que al atender una llamada telefónica se le olvidó que había dejado en la camilla a Mr. Smith (fig. 1), que presentaba dolor lumbar y en la pierna y que se había quedado acostado en la camilla, boca abajo. Pasados 10 min McKenzie se acordó’ de su paciente y al regresar y ver a Mr. Smith acostado en plena 
hiperextensión, sorprendido escucho': "por fin, después de tres semanas de tratamiento, me encuentro mucho mejor'. Esta historia, en realidad, describe un hecho que ha tenido una gran repercusión en el manejo de pacientes con dolor lumbar crónico, aunque McKenzie se fundamenta en otros datos más científicos para desarrollar su método. Experimenta el efecto de posiciones sostenidas y movimientos repetidos al final del rango de movimiento que desencadenan la sintomatología. Esto le permite plantear un sistema de clasificación de pacientes en función de las respuestas a diferentes posiciones y movimientos repetidos. Estos hallazgos están recogidos en varios libros ${ }^{2-5}$.

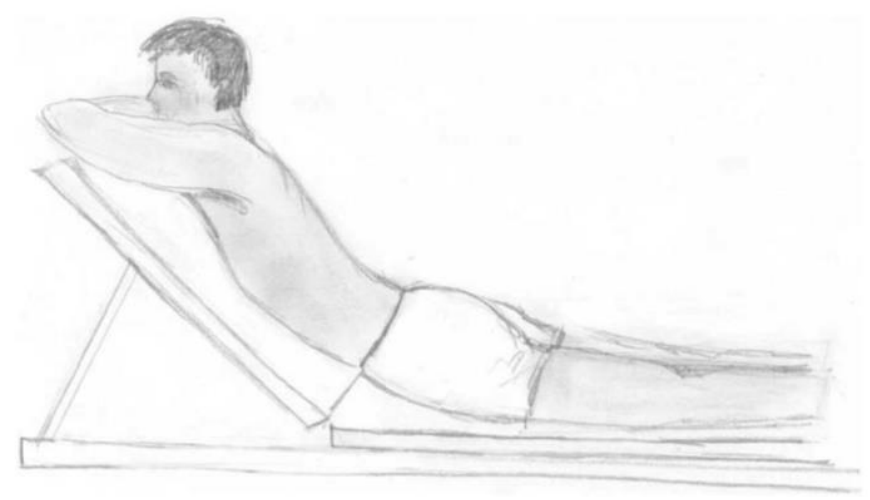

Figura 1 Posición de hiperextensión clásica de McKenzie.

En el apartado de valoración, uno de los aspectos más novedosos ha sido plantear que los movimientos simples (pedir al paciente que se mueva una sola vez, por ejemplo, a la flexión o a la extensión) no proveen una información suficiente para obtener una valoración meca ́nica adecuada ni para establecer la estrategia terapéutica pertinente. Es sólo con los movimientos repetidos o posturas mantenidas cuando los síntomas se presentan de una forma clara para ser interpretados. Una de las mayores contribuciones de McKenzie al abordaje de trastornos musculoesqueléticos ha sido demostrar el gran valor de los movimientos repetidos y posturas mantenidas al final del rango de movimiento, mientras se monitorizan los síntomas (centralización/periferalización) y las respuestas mecánicas (aumento o disminución de la movilidad) a dichos movimientos y/o posturas. La clave reside en no aislar la valoración del contexto cotidiano al que se tiene que enfrentar el paciente. Se tiene en cuenta la repetición de los movimientos y el tiempo de mantenimiento suficiente en determinadas posturas (por ejemplo, la sedestación), en coherencia con las solicitudes biomecánicas de la vida diaria del paciente. Una vez explorados los movimientos en cada dirección hasta el final del rango de movimiento y repetidas veces, se detecta cuál es el movimiento que elicita la respuesta más beneficiosa. Esto se corresponde con la preferencia direccional. Los términos de preferencia direccional, centralización/periferalización son términos específicos del método. La centralización hace referencia a que la realización de ciertos movimientos repetidos o posiciones mantenidas provocan que los síntomas cambien su localización 'n y se refieran más proximales, desplazándose hacia la línea media de la columna desde la periferia. La exploración del paciente implica encontrar la preferencia direccional. Dicha preferencia direccional se refiere a aquel movimiento o a aquella posición que provoca la centralización, la disminución e incluso la abolición de los síntomas, a la vez que permite una mejoría en los signos mecánicos (especialmente, la mejora de la amplitud de movimiento). La periferalización hace referencia a que otros movimientos o posturas, normalmente en la dirección opuesta a la que se logra la centralización, causan dolor, que se refiere distalmente, lejos de la línea media de la columna o hacia miembros inferiores ${ }^{2}$ (fig. 2). 


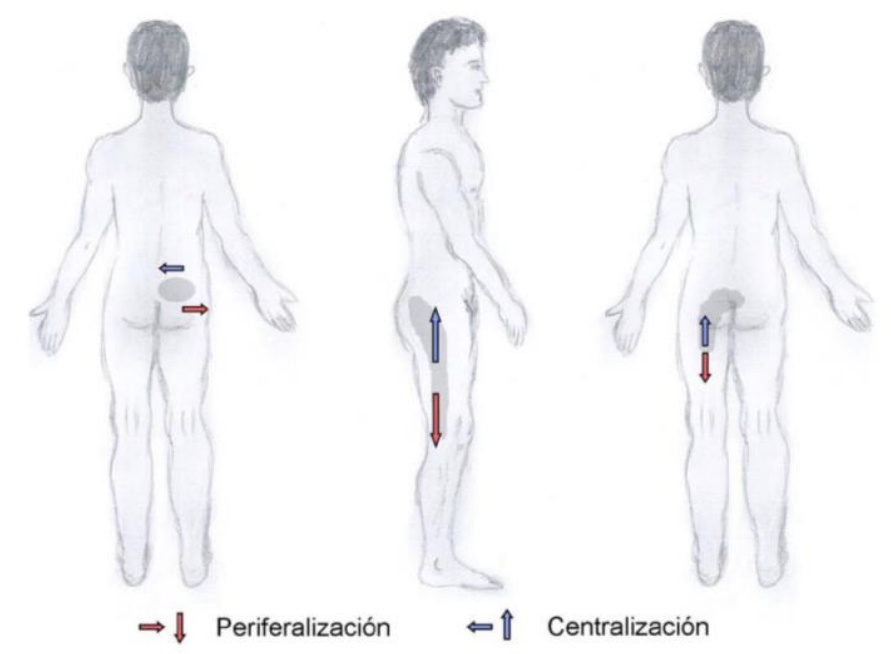

Figura 2 Dirección de los fenómenos de centralización y periferalización.

En el apartado terapéutico una de las aportaciones más importantes de este autor ha sido el planteamiento de una filosofía de autotratamiento. McKenzie es uno de los autores que más ha contribuido a revolucionar la forma de abordar los pacientes con dolor de espalda al darles un papel activo en su recuperación. Plantea como primera opción terapéutica el uso de fuerzas generadas por el propio paciente, justificando que, si el paciente es capaz de resolver su problema usando un programa regular de ejercicios y con consejos sobre cómo evitar los malos hábitos posturales, podrá' hacerse más independiente de otros tratamientos más pasivos. En realidad, McKenzie ha propuesto el enfoque de hands-off ("manos fuera") en el que se evita prácticamente la palpación durante el proceso de valoración y durante el tratamiento.

El autotratamiento incluiría, de forma genérica, tres fases: 1) demostrar y educar a los pacientes acerca del efecto beneficioso de determinadas posturas y movimientos al final de la amplitud, sobre sus síntomas, así como considerar también el efecto perjudicial y agravante de movimientos y posturas en la dirección opuesta a los primeros; 2) educar a los pacientes sobre cómo mantener la reducción de los síntomas e incluso cómo hacer que desaparezcan, y 3) educar a los pacientes sobre cómo restablecer la función de la columna lumbar sin que aparezca dolor. El paciente, a través de las indicaciones del fisioterapeuta y a través de su propia experiencia obtenida de las recomendaciones, asumirá' cada vez más un papel activo en su recuperación y en la prevención de su dolor lumbar. Los principales beneficios que supone el capacitar a la persona para el automanejo de su problema de salud son que se hace un uso más eficiente de los recursos limitados, se aumenta la implicación del paciente en el proceso de recuperación, se actúa directamente sobre las posibles causas del dolor (por ejemplo, incorporando hábitos biomecánicos correctos) y se facilita el manejo a largo plazo. Enfoque que, por otra parte, recomiendan diferentes revisiones sistemáticas y guías de práctica clínica ${ }^{6-9}$.

Propuesta de clasificación diagnostica de pacientes con dolor lumbar: el método de exploración estandarizado según McKenzie incluye una anamnesis exhaustiva y una exploración física (basada fundamentalmente en la respuesta a movimientos repetidos) que permiten al fisioterapeuta plantear una clasificación diagnostica provisional del paciente. Esta propuesta no busca tanto encontrar cuál es la estructura anatómica que causaría el dolor del paciente sino, más bien, cuál es el mecanismo lesional, es decir, que' es lo que desencadena dolor al paciente.

Según el MDT, los pacientes podrían clasificarse bajo 3 síndromes mecánicos: síndrome de desarreglo, síndrome de disfunción o síndrome postural (tabla 1). Recientemente, se ha incorporado un apartado de "otros", entre los que se incluye el síndrome de atrapamiento de la raíz nerviosa, patrón de dolor mecánico inconcluso y dolor no mecánico. Hay varios artículos publicados sobre esta propuesta de clasificación ${ }^{10-14}$ que han demostrado que la proporción de pacientes que pueden ser clasificados con este método, generalmente, es alto. Por ejemplo, se consiguió ${ }^{\prime}$ clasificar el $83 \%$ de 607 pacientes a partir de la clasificación de McKenzie entre fisioterapeutas con experiencia en el método, con el $78 \%$ de los pacientes clasificados como síndrome de desarreglo ${ }^{15}$. 
Tabla 1 Hallazgos genéricos de los 3 síndromes mecánicos

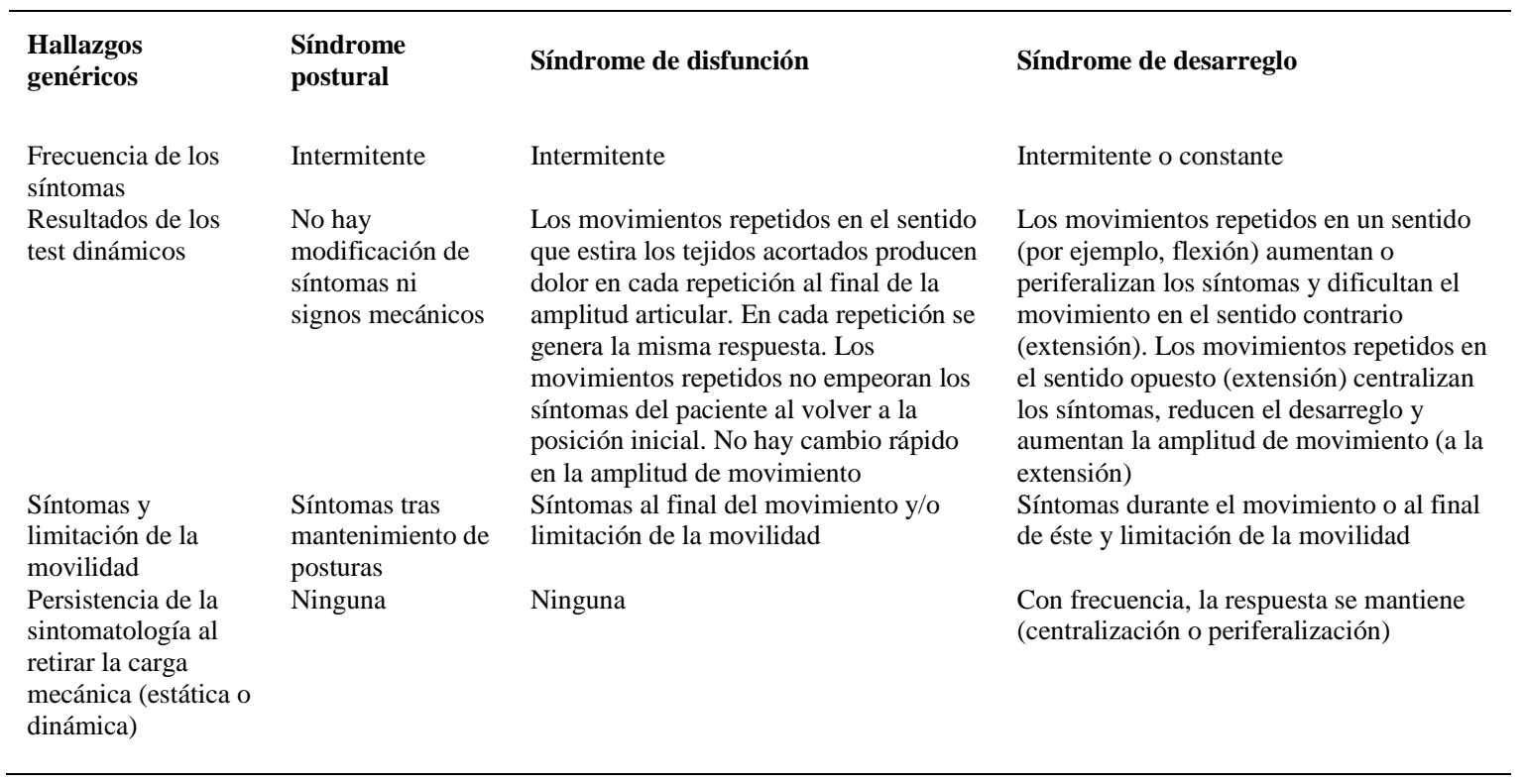

Los resultados de Kilpikoski ${ }^{16}$ también han sugerido que la fiabilidad interobservador para la clasificación de pacientes con dolor lumbar en los 3 síndromes parece ser alta cuando los fisioterapeutas tienen formación específica en el método (kappa=0,7).

Resultados similares encontró ${ }^{\prime}$ Razmjou ${ }^{12}$, que demostró una fiabilidad interobservador sustancial (kappa=0,7) para clasificar a los pacientes según los síndromes de McKenzie. Para identificar pacientes con síndrome de desarreglo la fiabilidad fue incluso superior (kappa=0,96).

Hefford $^{17}$, en un estudio observacional con 321 pacientes, encontró que el 77,9\% fue clasificado como síndrome de desarreglo reductible y la preferencia direccional predominante fue hacia la extensión. Sólo se tuvo en cuenta la primera sesión y los pacientes presentaban dolor lumbar, cervical o dorsal.

El síndrome de desarreglo está directamente relacionado con la patología del disco intervertebral. El elemento distintivo es que en él ocurre el fenómeno de centralización, generalmente en el sentido de la limitación del movimiento. McKenzie clasificó los desarreglos en tres tipos y a su vez en 7 categorías. Se denominan según la dirección en la que ha migrado el núcleo pulposo (y que mejor explica la respuesta sintomática y mecánica del paciente a las cargas mecánicas). El desarreglo n $.^{\circ} 1 \mathrm{y} \mathrm{n} .^{0} 2$ son desarreglos $^{\circ}$ posteriores, resultado de una migración posterocentral del núcleo pulposo. Los desarreglos numerados del 3 al 6 son desarreglos posterolaterales, resultado de una migración posterolateral del núcleo pulposo. El desarreglo n. ${ }^{\circ} 7$ es de tipo anterior, resultado de la migración anterior del núcleo pulposo.

En el síndrome de disfunción el dolor es producido por una deformación mecánica de tejidos blandos que ya están estructuralmente dañados. El daño de estos tejidos pudo haber sido por un traumatismo y/o por procesos inflamatorios previos o bien por los procesos degenerativos, etc., que pueden haber provocado, a su vez, contracturas, cicatrices, adherencias, etc. En definitiva, acortamientos adaptativos. El dolor es percibido cuando estos tejidos anormales son sometidos a estiramiento o compresión máxima, como ocurre en los rangos máximos de movimiento. Hay muchos modelos de pacientes que podrían presentar un síndrome de disfunción tal como lo plantea McKenzie. Por ejemplo:

a) Informático/a de 35 años que está $8 \mathrm{~h}$ al día en una posición de cabeza adelantada (síndrome postural) y que a la larga desarrollará un acortamiento adaptativo de diferentes tejidos blandos (musculares, neurales, etc.).

b) Un conductor de camión de 40 años que está 10 h al día en una posición de sentado hundido (síndrome postural), que a la larga desarrolla una incapacidad para alcanzar una lordosis lumbar normal en posición de pie debido al acortamiento progresivo de diferentes tejidos blandos.

c) Pacientes que han sufrido un accidente de coche o cirugía o, incluso, personas mayores que van sufriendo una pérdida progresiva de movilidad casi simétrica en todas direcciones.

La característica común es una alteración del tejido conectivo (consecuencia de microtraumatismos o macrotraumatismos) que cicatriza y/o se adapta al acortamiento. Esto hace más difícil identificar la 
estructura que está causando la disfunción, pero no impide elegir la estrategia terapéutica (de remodelación) apropiada. Una de las características clave de este síndrome es la presencia de dolor solamente al final de la amplitud máxima del movimiento articular y nunca durante, tanto al movimiento simple como a los movimientos repetidos. La intensidad y la localización del dolor es similar con cada repetición; el dolor además cede después de unos minutos y vuelve a su estado de dolor inicial. A diferencia del síndrome de desarreglo, no se producen cambios rápidos en los síntomas y signos mecánicos como resultado de movimientos repetidos. McKenzie describe el síndrome de adherencia de raíz 'z nerviosa como un tipo particular de disfunción en el que el dolor sería desencadenado por la puesta en tensión de la raíz nerviosa y/o su tejido cicatrizal.

En el síndrome postural el dolor es producido por deformación mecánica (nocicepción mecánica) de cualquiera de los tejidos normales y sanos de la columna vertebral cuando son sometidos a cargas estáticas prolongadas. La causa más frecuente de este síndrome son los malos hábitos posturales, adoptados desde muy temprana edad, sobre todo, la posición de sentado hundido, las posiciones de flexión de tronco y la posición de pie prolongada. Es un dolor de tipo intermitente, normalmente localizado en la línea media de la columna o simétrico. El dolor se alivia cuando se corrige la postura (especialmente con el restablecimiento de la lordosis lumbar). Habrá que diferenciar este síndrome de aquellos otros (disfunción o desarreglo) que pueden agravarse igualmente si los tejidos son sometidos a cargas estáticas prolongadas.

Aquellos pacientes que no puedan clasificarse bajo alguno de estos 3 síndromes después de varias sesiones con el MDT deberían ser clasificados bajo el epígrafe de "otros síndromes".

Enfoque terapéutico en función de la clasificación: en el síndrome de desarreglo el objetivo principal es centralizar el dolor y restablecer la movilidad lumbar. Para resolver el desarreglo del disco McKenzie se basa en el modelo dinámico interno del disco. Se fundamenta en que el disco puede ser influenciado por los movimientos y posturas para resolver sus propias alteraciones $\mathrm{y}$, por lo tanto, responder positivamente a un tratamiento de tipo mecánico como el que propone ${ }^{18-20}$.

En el síndrome de disfunción, como los síntomas se producen por la deformación mecánica de tejidos que están estructuralmente dañados, el objetivo será seleccionar y aplicar el movimiento en la dirección apropiada que es aquella que reproduce el dolor del paciente. Esto nos indica que el tejido en disfunción está siendo remodelado y reparado, por lo que el dolor ira' disminuyendo gradualmente.

En el síndrome postural, el dolor se alivia a partir de la corrección postural. El objetivo principal es educar al paciente en la importancia de la corrección de las posturas inadecuadas (sobre todo, la postura de sentado) al tiempo que experimenta los efectos beneficiosos sobre el dolor de tales correcciones.

El MDT propone para cada síndrome una progresión específica de las estrategias de tratamiento, pero que en ningún caso es una prescripción genérica y estándar para cada síndrome, sino que el tratamiento se plantea de forma individualizada en función de las respuestas obtenidas durante la exploración. Los dos elementos que guían la selección de las estrategias terapéuticas son la preferencia direccional y la aplicación de fuerzas progresivas.

La preferencia direccional puede ser hacia la extensión (es la preferencia direccional más frecuente en el síndrome de desarreglo), hacia la flexión, hacia la inclinación o una combinación de fuerzas sagitales y laterales. El tratamiento consiste en que el paciente realice de forma repetida ejercicios hasta el final del rango de movimiento en la dirección que coincide con la preferencia direccional. El tratamiento puede consistir igualmente en el mantenimiento de posturas en la preferencia direccional durante un cierto tiempo, por ejemplo, hacia la extensión como la que se muestra en la figura 1. El tratamiento puede consistir igualmente en el mantenimiento de posturas en la preferencia direccional durante un cierto tiempo. Como esta preferencia no es estable durante todo el tratamiento, habrá que ir adecuándola a la evolución del cuadro clínico. Por ejemplo, habrá pacientes que tienen una preferencia direccional hacia la extensión, que al principio la toleren bien de pie y no en prono o viceversa.

La aplicación de fuerzas progresivas describe la escalada de las estrategias de carga terapéutica, desde los movimientos centrados y realizados por el paciente a las técnicas pasivas realizadas por el fisioterapeuta (estas últimas sólo cuando sean estrictamente necesarias). Este concepto de progresión no implica necesariamente que tengamos que empezar siempre en el mismo punto de partida.

Basándose en el enfoque de hand-off, McKenzie sólo aplica técnicas pasivas cuando las técnicas activas no resuelven el problema o no son suficientes. En estos casos, el MDT contempla técnicas de presión al final del rango de movimiento (por ejemplo, el paciente hace el ejercicio de extensión en decúbito-prono y el fisioterapeuta aplica presión al final del movimiento en un determinado nivel articular que presente desarreglo o disfunción) (fig. 3). También puede aplicar técnicas de movilización y/o manipulación que se aplicarán igualmente según la preferencia direccional. Aún en aquellos casos que requieran la aplicación de fuerzas pasivas para obtener la centralización y el alivio de los síntomas, una vez obtenidos éstos, se darán al paciente recomendaciones para el autotratamiento bajo la supervisión del fisioterapeuta. 

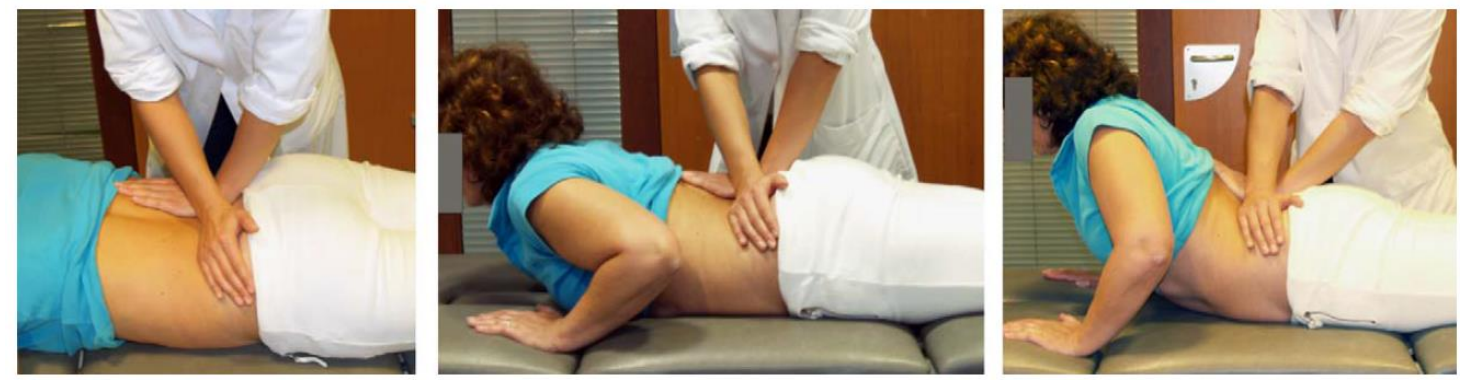

Figura 3 Automovilización en extensión con ayuda del fisioterapeuta.

El rol que debe asumir el fisioterapeuta debe ser fundamentalmente como evaluador, clasificador y educador.

Evidencia sobre la eficacia del método: dentro de este apartado se hará referencia a las guías de práctica clínica, revisiones sistemáticas, estudios específicos sobre el fenómeno de centralización y ensayos clínicos randomizados.

Según May ${ }^{1}$, la investigación sobre el método McKenzie se inicia en 1990 con la publicación de un estudio sobre fiabilidad diagnóstica ${ }^{11}$, un ensayo clínico randomizado ${ }^{21}$ y un estudio sobre el concepto de centralización ${ }^{22}$. Desde entonces la literatura científica sobre el método ha seguido creciendo y actualmente existen estudios tanto sobre el componente de intervención como sobre la fiabilidad y el valor pronóstico del sistema de valoración.

Cuando en las guías de práctica clínica se hace referencia al método McKenzie, hay que destacar que en la mayoría de los casos se refieren casi de forma exclusiva al componente terapéutico y, en ocasiones, lo igualan casi exclusivamente a ejercicios de extensión ${ }^{1}$. Teniendo en cuenta estas limitaciones, el método McKenzie ha sido recomendado en 4 guías de práctica clínica ${ }^{23-26}$ (tabla 2).

Tabla 2 Método McKenzie y guías de práctica clínica

\begin{tabular}{|c|c|c|}
\hline DIHTA $^{23}$ & $\begin{array}{l}\text { - Método diagnóstico e indicador pronóstico } \\
\text { - Tratamiento del dolor lumbar agudo y } \\
\text { crónico }\end{array}$ & $\begin{array}{l}\text { - Nivel B } \\
\text { - Nivel C }\end{array}$ \\
\hline $\operatorname{ASSSM}^{25}$ & $\begin{array}{l}\text { - Dolor lumbar agudo (de } 0 \text { a } 4 \text { semanas) } \\
\text { - Dolor lumbar subagudo (de } 4-12 \text { semanas) } \\
\text { - Dolor lumbar crónico ( } 412 \text { semanas) }\end{array}$ & $\begin{array}{l}\text { - Baja evidencia científica } \\
\text { - Moderada evidencia científica } \\
\text { - Baja evidencia científica }\end{array}$ \\
\hline Poitrasa $^{27}$ & $\begin{array}{l}\text { - Dolor lumbar agudo } \\
\text { - Dolor lumbar subagudo } \\
\text { - Dolor crónico }\end{array}$ & $\begin{array}{l}\text { - Nivel de evidencia bajo (C) } \\
\text { - Nivel de evidencia moderado (B) } \\
\text { - Nivel de evidencia bajo (C) }\end{array}$ \\
\hline
\end{tabular}

ASSSM: Agence de la Sante' et des Services Sociaux de Montreal; DIHTA: Danish Institute for Health Technology Assessment.

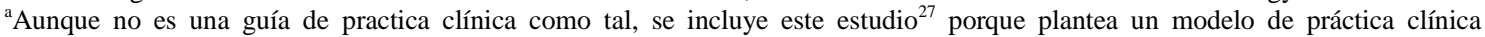
interdisciplinario para el manejo de pacientes con dolor lumbar crónico, en el que se hace referencia al método McKenzie. 
En tres revisiones sistemáticas se concluye que hay evidencia limitada del método McKenzie relacionada con el dolor de espalda crónico. Clare ${ }^{28}$ revisó 6 ensayos clínicos (5 sobre dolor lumbar) y los resultados demostraron que para pacientes con dolor de espalda, a corto plazo (seguimiento de 3 meses o inferior), el método McKenzie tenía mejores resultados en términos de alivio de dolor y mejora de la incapacidad funcional que otros tratamientos estándares (como, por ejemplo, un folleto educacional, ejercicio general, medicación, masaje no específico, ejercicios de fortalecimiento y estiramiento o movilización pasiva). Sin embargo, los datos parecen ser insuficientes para valorar la eficacia del método a largo plazo. Machado ${ }^{29}$ concluye que hay alguna evidencia de que el método McKenzie es más eficaz que el tratamiento pasivo en pacientes con dolor de espalda agudo, aunque la magnitud de la diferencia sugiere la ausencia de efectos relevantes clínicamente, y que hay evidencia limitada para el uso del método McKenzie en pacientes con dolor lumbar crónico. Cook ${ }^{30}$, en otra revisión sistemática (que incluyó 5 estudios, 4 de los cuales utilizaron el método McKenzie, todos ellos con una calidad metodológica alta), evaluó la evidencia del ejercicio, con la particularidad de que los pacientes habían sido clasificados previamente en función de la respuesta sintomática. Se incluyeron pacientes con síntomas de duración variable (algunos crónicos y la mayoría subagudos). Encontraron que un programa de ejercicios dirigido en función de la respuesta sintomática del paciente consiguió mejores resultados que en los grupos control. Los autores mencionan la necesidad de estudios en los que se comparen diferentes formas de clasificar a los pacientes con dolor lumbar.

Entre otras revisiones no exclusivamente centradas en el MDT (revisiones inespecíficas) se encuentran las realizadas por Slade ${ }^{31,32}$. En la primera revisión ${ }^{31}$, centrada en los ejercicios de fortalecimiento, concluye que éstos son más efectivos para la reducción del dolor y la mejora de la función que el no-ejercicio. Sin embargo, cita expresamente que para algunos pacientes con dolor lumbar, los ejercicios de McKenzie parecen ser más beneficiosos que los programas de fortalecimiento. En la revisión más reciente se concluye que en pacientes con dolor lumbar no específico hay fuerte evidencia de que los ejercicios "de baja carga" (entre los que incluye el método McKenzie y yoga) comparado con no-ejercicio son efectivos para mejorar el dolor y la funcionalidad ${ }^{32}$. Cuando estos ejercicios "de baja carga" se comparan con otros tipos de ejercicios (de potenciación y de estabilización) los efectos parecen similares32 (tabla 3).

Tabla 3. Revisiones sistemáticas

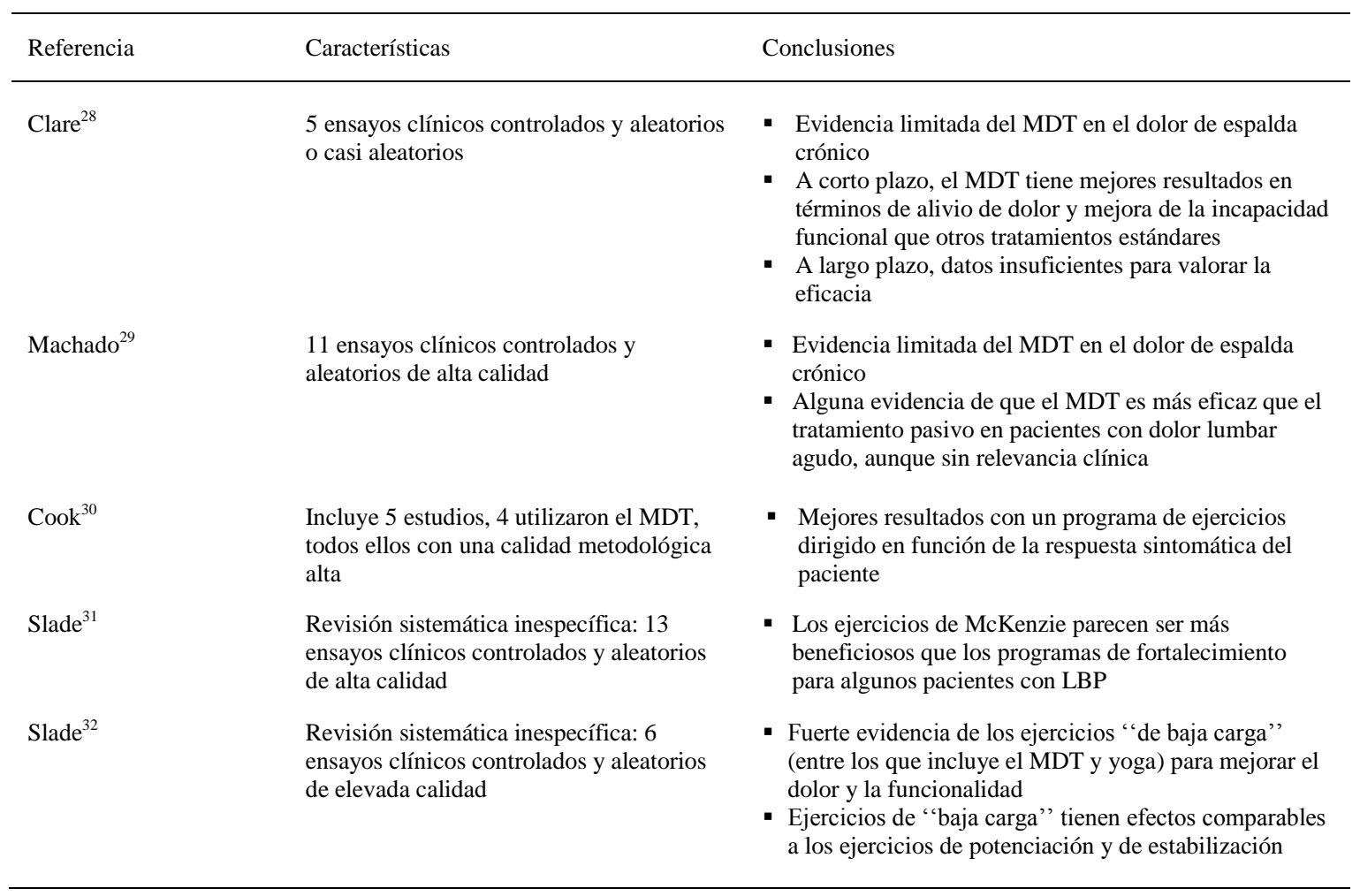

LBP: pacientes con dolor lumbar; MDT: Method of Mechanical Diagnosis and Therapy 'método de diagnóstico y tratamiento mecánico'. 
En relación con el componente de evaluación del método McKenzie (fenómeno de centralización) existen dos revisiones sistemáticas relevantes ${ }^{33,34}$ (tabla 4). La primera ${ }^{33}$ concluyó que la centralización de los síntomas es un fenómeno clínico común y que, con una formación apropiada, puede ser evaluada con una gran fiabilidad. Además, tiene importantes implicaciones terapéuticas y de pronóstico; cuando aparece está asociada de forma consistente con buenos resultados. El estudio concluye, asimismo, que este fenómeno debería valorarse durante la exploración del paciente y utilizarse como guía para seleccionar las estrategias terapéuticas más apropiadas. Como dato complementario a esta revisión hay que señalar que Werneke ${ }^{35}$ demostró que un alto porcentaje de pacientes no centralizaba los síntomas en la primera visita, pero si' aparecía en las sesiones posteriores. Encontró que la centralización sólo ocurría en un 3,2\% de una población con dolor lumbar agudo/subagudo durante la exploración inicial, pero que este porcentaje se elevaba hasta el $63,8 \%$ en varios días. En la segunda revisión ${ }^{34}$, que analiza los diferentes métodos de valoración de dolor lumbar inespecífico, se encuentra que la evaluación basada en la respuesta de los síntomas a movimientos repetidos cuenta con una evidencia moderada de fiabilidad alta (criterio establecido: kappa $\geq 0,7$ ).

Tabla 4. Revisiones sistemáticas sobre el fenómeno de centralización

\begin{tabular}{|c|c|c|}
\hline Referencia & Objetivo & Conclusiones \\
\hline $\mathrm{Aina}^{33}$ & Estudios sobre centralización (14 estudios revisados) & $\begin{array}{l}\text { - El } 70 \% \text { de prevalencia de centralización en } 731 \\
\text { pacientes con dolor de espalda subagudo y el } \\
52 \% \text { en } 325 \text { pacientes con dolor de espalda } \\
\text { crónico } \\
\text { - Asociación consistente con buen pronóstico } \\
\text { - Valor kappa de } 0,51-1,0\end{array}$ \\
\hline May $^{34}$ & $\begin{array}{l}\text { Revisión de } 48 \text { estudios sobre fiabilidad de diferentes } \\
\text { pruebas de exploración de pacientes con dolor lumbar } \\
\text { inespecífico (incluyen valoración de la respuesta de } \\
\text { dolor a movimientos repetidos) }\end{array}$ & $\begin{array}{l}\text { Evidencia moderada sobre fiabilidad alta (kappa } \geq \\
0,7 \text { ) para la valoración de la respuesta de los } \\
\text { síntomas a los movimientos repetidos }\end{array}$ \\
\hline
\end{tabular}

Se llevaron a cabo cuatro estudios posteriores a las revisiones citadas acerca del fenómeno de centralización $^{36-39}$. Laslett ${ }^{36}$ encontró una alta especificidad (del 80 al 100\%) de la centralización en relación con la existencia de discografía positiva. Cuando se producía el fenómeno de centralización durante la valoración inicial, la probabilidad de una discografía positiva era muy alta y sería razonable el hacer un diagnóstico de dolor discogénico. Los resultados reflejaron que el fenómeno de centralización (por su modesta sensibilidad) no permite ser usado como un instrumento de screening para seleccionar pacientes para discografía. Sin embargo, su alta especificidad posibilita que el fisioterapeuta pueda hacer el diagnóstico de dolor discogénico sin recurrir al examen por discografía invasiva. Cuando el paciente presentaba una severa incapacidad funcional (medida con escala de Roland-Morris) se incluía un factor de confusión que hacía que la especificidad de la centralización disminuyera, de tal manera que en estos casos la centralización era sugestiva de dolor discogénico, pero no lo determinaba con tan alta probabilidad.

Skytte ${ }^{37}$ identificó que, tanto a corto como a largo plazo, los pacientes que centralizaron sus síntomas tuvieron significativamente mejores resultados de dolor e incapacidad. En cambio, los no-centralizadores tuvieron 6 veces más probabilidad de recibir tratamiento quirúrgico que los centralizadores.

Berthelot $^{38}$ en una revisión de la literatura médica destaca varias limitaciones del fenómeno de centralización: la falta de criterios estandarizados para definirlo (especialmente en relación con el cambio mínimo que debe darse en la localización del dolor y al tiempo en el que ocurre), que dicho fenómeno no es suficientemente especifico de dolor discogénico como para obviar realizar otras pruebas diagnósticas al paciente, y que hay pacientes que son incapaces de tolerar la valoración propuesta por McKenzie y que esto podría limitar su uso en pacientes mayores.

Werneke $^{39}$ concluye que el uso clínico de una definición estándar de centralización facilita la clasificación, manejo e interpretación de los resultados en pacientes con dolor lumbar y cervical.

En referencia a la importancia de la centralización con respecto a otros factores predictores de la evolución del dolor de espalda se han realizado diversos estudios. Wernecke y Hart ${ }^{40}$ determinaron que los dos factores predictores más importantes de dolor crónico e incapacidad fueron la no-centralización y 
el dolor en la pierna. Los mismos autores, en un estudio posterior ${ }^{41}$, concluyen que la no-centralización puede ser un indicador de comportamiento de enfermedad y no un hallazgo físico dada su asociación con muchos signos comportamentales. Por tanto, cuando ocurre la no-centralización debería considerarse el realizar un cribado psicosocial durante la evaluación inicial. George ${ }^{42}$ encontró que tanto el fenómeno de no-centralización como las creencias de miedo-evitación sobre el trabajo predecían una mayor incapacidad. Además, la no-centralización predecía mayor intensidad de dolor a los 6 meses, aunque los resultados se referían solamente a pacientes con dolor lumbar agudo clasificados para ejercicios específicos.

Son cuatro los ensayos clínicos randomizados más relevantes en el tratamiento del dolor lumbar crónico usando el método McKenzie ${ }^{43-47}$ con poblaciones diferentes en cuanto a duración de la sintomatología y con tratamientos distintos (ejercicios de potenciación, de estabilización, ejercicios en diferentes direcciones a la preferencia direccional o sesiones educacionales basadas en los principios cognitivoconductuales). Los resultados son claramente favorables, a corto plazo, para el grupo tratado con el método McKenzie en uno de los estudios $^{43}$ y los resultados son igualmente eficaces para los tratamientos comparados en los otros tres ensayos ${ }^{44-47}$.

Powers ${ }^{48}$ comparó el efecto de una sesión de movilización posteroanterior y de un ejercicio de pressup (ejercicio dinámico de extensión de columna en decúbito-prono, a través de flexoextensión de codos, manteniendo la pelvis apoyada) y obtuvo un efecto inmediato y similar en cuanto a reducción dolor e incremento de la movilidad a la extensión lumbar (tabla 5). 
Tabla 5. RCT sobre el método McKenzie en el dolor lumbar crónico

\begin{tabular}{|c|c|c|c|c|}
\hline Referencia & Grupos (n) & Seguimiento & Mediciones & Resultados \\
\hline Long $^{43}$ & $\begin{array}{l}\text { 1. Ejercicios en la PD }(\mathrm{n}=80) \\
\text { 2. Ejercicios en la dirección opuesta a la } \\
\mathrm{PD}(\mathrm{n}=70) \\
\text { 3. Ejercicios no específicos }(\mathrm{n}=80)\end{array}$ & $\begin{array}{l}230 \text { pacientes con dolor agudo, subagudo y crónico } \\
\text { que presentaban una PD (a la extensión [83\%], a la } \\
\text { flexión [7\%] o a la traslación [10\%]) }\end{array}$ & $\begin{array}{l}\text { - Localización e intensidad del dolor } \\
\text { - Medicación } \\
\text { - Incapacidad funcional (RDQ) } \\
\text { - Depresión } \\
\text { - Interferencias del dolor en las actividades } \\
\text { laborales y de casa }\end{array}$ & $\begin{array}{l}\text { Mejoras significativas a favor de los ejercicios } \\
\text { en la PD (método McKenzie) en todos las } \\
\text { mediciones realizadas }\end{array}$ \\
\hline Petersen $^{44,45}$ & $\begin{array}{l}\text { 1. McKenzie }(n=132) \\
\text { 2. Ejercicios de potenciación }(n=128)\end{array}$ & $\begin{array}{l}\text { - } 180 \text { pacientes completaron el tratamiento }(70 \%) \\
\text { Al final del tratamiento ( } 8 \text { semanas) } \\
\text { - A los } 2 \text { y a los } 8 \text { meses } \\
\text { - } \mathrm{Al} \text { año (Petersen, 2007) }\end{array}$ & $\begin{array}{l}\text { - Dolor } \\
\text { - Incapacidad funcional }\end{array}$ & Resultados igualmente eficaces \\
\hline Miller $^{46}$ & $\begin{array}{l}\text { 1. McKenzie }(n=15) \\
\text { 2. Ejercicios de estabilización }(n=15)\end{array}$ & $\begin{array}{l}\text { - Al final del tratamiento (6 semanas) } \\
29 \text { pacientes completaron el tratamiento }(97 \%)\end{array}$ & $\begin{array}{l}\text { - Incapacidad funcional } \\
\text { - Dolor } \\
\text { - Test de SLR }\end{array}$ & $\begin{array}{l}\text { No hubo diferencias significativas entre los dos } \\
\text { grupos }\end{array}$ \\
\hline Moffet $^{47}$ & $\begin{array}{l}\text { 1. Tratamiento McKenzie }(\mathrm{n}=161), 4-5 \\
\text { sesiones } \\
\text { 2. Sesiones educacionales basadas en } \\
\text { los principios cognitivo-conductuales } \\
(\mathrm{n}=154), 3 \text { sesiones }\end{array}$ & $\begin{array}{l}\text { - } 315 \text { pacientes ( } 219 \text { con dolor lumbar y } 96 \text { con } \\
\text { dolor cervical) } \\
\text { - A las } 6 \text { semanas (McKenzie, } n=133 / \text { educ, } \\
\mathrm{n}=126 \text { ) } \\
\text { - } \mathrm{A} \text { los } 6 \text { meses (McKenzie, } \mathrm{n}=123 / \text { educ, } \mathrm{n}=117 \text { ) } \\
\text { - A los } 12 \text { meses (McKenzie, } \mathrm{n}=132 / \mathrm{educ}, \mathrm{n}=111 \text { ) } \\
30 \text { adultos diagnosticados de dolor lumbar }\end{array}$ & $\begin{array}{l}\text { - Actividad-evitación (TSK) } \\
\text { - Incapacidad funcional lumbar (RDQ) } \\
\text { - Incapacidad funcional cervical (NPQ) } \\
\text { - Calidad de vida SF-12 } \\
\text { - Otros cuestionarios sobre dolor, ansiedad, etc. }\end{array}$ & $\begin{array}{l}\text { No hubo diferencias significativas entre los dos } \\
\text { grupos, excepto que el método McKenzie fue } \\
\text { ligeramente más efectivo a los } 6 \text { meses, para } \\
\text { reducir el comportamiento de evitación a la } \\
\text { actividad, y que a las } 6 \text { semanas la satisfacción } \\
\text { del paciente fue mayor con este método. } \\
\text { Resultados similares }\end{array}$ \\
\hline Powers ${ }^{8}$ & $\begin{array}{l}\text { 1. Un ejercicio de press-up }{ }^{\mathrm{a}} \\
\text { 2. } 2 \text { movilizaciones posteroanterior }\end{array}$ & $\begin{array}{l}30 \text { adultos diagnosticados de dolor lumbar } \\
\text { inespecífico }\end{array}$ & $\begin{array}{l}\text { - Intensidad del dolor } \\
\text { - Amplitud del movimiento con RMN dinámica }\end{array}$ & Resultados similares \\
\hline
\end{tabular}

Educ: sesiones educacionales basados en los principios cognitivo-conductuales; PD: preferencia direccional; RMN: resonancia magne'tica nuclear; SLR: Straight Leg Raising 'test de elevación de la pierna extendida'; TSK: Tampa Scale for Kinesiophobia.

a Ejercicio de press-up (ejercicio de flexiones de brazo en decúbito-prono) 


\section{Conclusiones}

El método McKenzie presenta una eficacia similar, a corto plazo, a los ejercicios de estabilización y una tendencia favorable frente a ejercicios de fortalecimiento en el dolor lumbar.

El fenómeno de centralización tiene un valor pronóstico positivo aunque existe cierta controversia sobre su especificidad como indicador de dolor discogénico.

La fiabilidad como método de exploración es alta y favorece un abordaje terapéutico más específico y exitoso. Sin embargo, se hace necesario realizar una estandarización y unificación de criterios, como la definición operativa del fenómeno de centralización. Por otra parte, dicha exploración podría no ser aplicable a todo tipo de pacientes.

\section{Conflicto de intereses}

Declaramos no tener ningún conflicto de interés.

\section{Bibliografía}

1. May S, Donelson R. Evidence informed management of chronic low back pain with the McKenzie method. Spine J. 2008;8: 134-141.

2. McKenzie R, May S. The lumbar spine: Mechanical diagnosis and therapy, Vol. 1-2. 2 ed. Waikanae, New Zealand: Spinal Publications; 2003.

3. McKenzie R. The lumbar spine: Mechanical diagnosis and therapy. Waikanae, New Zealand: Spinal Publications; 1981.

4. McKenzie R, May S. The human extremities: Mechanical diagnosis and therapy. Waikanae, New Zealand: Spinal Publications; 2000.

5. McKenzie R, May S. The cervical and thoracic spine: Mechanical diagnosis and therapy, Vol. 1-2. Waikanae, New Zealand: Spinal Publications; 2003.

6. Hilde G, Hagen KB, Jamtvedt G, Winnem MF. Advice to stay active as a single treatment for low back pain and sciatica. Cochrane Database of Systematic Reviews 2006. Art. N.o: CD003632. doi:10.1002/14651858.CD003632.pub2.

7. Chou R, Qaseem A, Snow V, Casey D, Cross Jr JT, Shekelle P, et al. Clinical guidelines: Diagnosis and treatment of low back pain: A joint clinical practice guideline from the American College of Physicians and the American Pain Society. Ann Intern Med. 2007;147:478-91.

8. Hilde G, Hagen KB, Jamtvedt G, Winnem M. Advice to stay active as a single treatment for low-back pain and sciatica. Cochrane Database of Systematic Reviews. 2007:CD003632.

9. Liddle SD, Gracey JH, Baxter GD. Advice for the management of low back pain: A systematic review of randomised controlled trials. Man Ther. 2007;12:310-27.

10. Clare HA, Adams R, Maher CG. Reliability of McKenzie classification of patients with cervical or lumbar pain. J Manipulative Physiol Ther. 2005;28:122-7.

11. Kilby J, Stigant M, Roberts A. The reliability of back pain assessment by physiotherapists, using a "McKenzie algorithm'. Physiotherapy. 1990;76:579-83.

12. Razmjou H, Kramer JF, Yamada R. Intertester reliability of the McKenzie evaluation in assessing patients with mechanical low-back pain. J Orthop Sports Phys Ther. 2000;30: 368-383.

13. Riddle DL, Rothstein JM. Intertester reliability of McKenzie0s classifications of the syndrome types present in patients with low back pain. Spine. 1993;18:1333-44.

14. Clare HA. Evaluation of the McKenzie method. Sidney: University of Sydney; 2005.

15. May S. Classsification by McKenzie mechanical syndromes: A survey of McKenzie-trained faculty. J Manipulative Physiol Ther. 2006;29:637-42.

16. Kilpikoski S, Airaksinen O, Kankaanpaa M, Leminen P, Videman T, Alen M. Interexaminer reliability of low back pain assessment using the McKenzie method. Spine. 2002;27:E207-14.

17. Hefford C. McKenzie classification of mechanical spinal pain: Profile of syndromes and directions of preference. Man Ther. 2008;13:75-81.

18. Alexander LA, Hancock E, Agouris I, Smith FW, MacSween A. The response of the nucleus pulposus of the lumbar intervertebral discs to functionally loaded positions. Spine. 2007;32:1508-12.

19. Edmondston SJ, Song S, Bricknell RV, Davies PA, Fersum K, Humphries P, et al. MRI evaluation of lumbar spine flexion and extension in asymptomatic individuals. Man Ther. 2000;5: 158-164.

20. Beattie PF, Brooks WM, Rothstein JM, Sibbitt Jr WL, Robergs RA, MacLean T, et al. Effect of lordosis on the position of the nucleus pulposus in supine subjects. A study using magnetic resonance imaging. Spine. 1994;19:2096-102.

21. Stankovic R, Johnell O. Conservative treatment of acute lowback pain. A prospective randomized trial: McKenzie method of treatment versus patient education in "mini back school". Spine. 1990;15:120-3.

22. Donelson R, Silva G, Murphy K. Centralization phenomenon. Its usefulness in evaluating and treating referred pain. Spine. 1990;15:211-3. 
23. Danish Institute for Health Technology Assessment: Low-back pain. Frequency, management and prevention from an HTA perspective Danish Health Technology Assessment. 1999;1.

24. Philadelphia Panel. Philadelphia panel evidence-based clinical practice guidelines on selected rehabilitation interventions for low back pain. Phys Ther. 2001;81:1641-74.

25. Agence de la santé et des services sociaux de Montreal. Quebec. Clinique des Lombalgies Interdiciplinaire en Premiere Ligne. Guide de pratique. Montreal, Quebec, Canada: 2006.

26. Mercer C, Jackson A, Hettinga D, Barlos P, Ferguson S, Greenhalh S. Exercise. In: Chartered Society of Physiotherapy. Clinical Guidelines for the physiotherapy management of persistent low back pain. London: CSP; 2006.

27. Poitras S, Rossignol M, Dionne C, Tousignant M, Truchon M, Arsenault B, et al. An interdisciplinary clinical practice model for the management of low-back pain in primary care: The CLIP Project. BMC Musculoskelet Disord. 2008;9:54.

28. Clare HA, Adams R, Maher CG. A systematic review of efficacy of McKenzie therapy for spinal pain. Aust J Physiother. 2004;50: 209-216.

29. Machado LA, De Souza MS, Ferreira PH, Ferreira ML. The McKenzie method for low back pain: A systematic review of the literature with a meta-analysis approach. Spine. 2006;31: E254-E262.

30. Cook C, Ramey K, Hegedus E. Physical therapy exercise intervention based on classifcation using the patient response method: A systematic review of the literature. J Man Manipulative Ther. 2005;13:152-62.

31. Slade SC, Keating JL. Trunk-strengthening exercises for chronic low back pain: A systematic review. J Manipulative Physiol Ther. 2006;29:163-73.

32. Slade SC, Keating JL. Unloaded movement facilitation exercise compared to no exercise or alternative therapy on outcomes for people with nonspecific chronic low back pain: A systematic review. J Manipulative Physiol Ther. 2007;30:301-11.

33. Aina A, May S, Clare H. The centralization phenomenon of spinal symptoms: A systematic review. Man Ther. 2004;9: 134-143.

34. May S, Littlewood C, Bishop A. Reliability of procedures used in the physical examination of non-specific low back pain: A systematic review. Aust J Physiother. 2006;52:91-102.

35. Werneke M, Hart DL. Discriminant validity and relative precision for classifying patients with non-specific neck and back pain by anatomic pain patterns. Spine. 2003;28:161-6.

36. Laslett M, O"berg B, Aprill CN, McDonald B. Centralization as a predictor of provocation discography results in chronic low back pain, and the influence of disability and distress on diagnostic power. Spine J. 2005;5:370-80.

37. Skytte L, May S, Petersen P. Centralization: Its prognostic value in patients with referred symptoms and sciatica. Spine. 2005;30:E293-9.

38. Berthelot JM, Delecrin J, Maugars Y, Passuti N. Contribution of centralization phenomenon to the diagnosis, prognosis and treatment of diskogenic low back pain. Joint Bone Spine. 2007;74:319-23.

39. Werneke MW, Hart DL, Resnik L, Stratford PW, Reyes A. Centralization: Prevalence and effect on treatment outcomes using a standardized operational definition and measurement method. J Orthop Sports Phys Ther. 2008;38:116-25.

40. Werneke M, Hart DL. Centralization phenomenon as a prognostic factor for chronic low back pain and disability. Spine. 2001;26:758-64.

41. Werneke M, Hart DL. Centralization: Association between repeated end-range pain responses and behavioral signs in patients with acute non-specific low back pain. J Rehabil Med. 2005;37:286-90.

42. George SZ, Bialosky JE, Donald DA. The centralization phenomenon and fear-avoidance beliefs as prognostic factors for acute low back pain; a preliminary investigation involving patients classified for specific exercise. J Orthop Sports Phys Ther. 2005;35:580-8.

43. Long A, Donelson R, Fung T. Does it matter which exercise? A randomized control trial of exercise for low back pain Spine. 2004;29:2593-602.

44. Petersen T, Kryger P, Ekdahl C, Olsen S, Jacobsen S. The effect of McKenzie therapy as compared with that of intensive strengthening training for the treatment of patients with subacute or chronic low back pain: A randomized controlled trial. Spine. 2002;27:1702-9.

45. Petersen T, Larsen K, Jacobsen S. One-year follow-up comparison of the effectiveness of McKenzie treatment and strengthening training for patients with chronic low back pain: Outcome and prognosis factors. Spine. 2007;32:2948-56.

46. Miller ER, Schenk Pj, Karnes JL, Rouselle JG. A comparison of the McKenzie approach to a specific spine stabilization program for chronic low back pain. J Man Manip Ther. 2005;13:103-12.

47. Moffett JK, Jackson DA, Gardiner ED, Torgerson DJ, Coulton S, Eaton S, et al. Randomized trial of two physiotherapy interventions for primary care neck and back pain patients: 'McKenzie' vs. brief physiotherapy pain management. Rheumatology (Oxford). 2006;45:1514-21.

48. Powers CM, Beneck GJ, Kulig K, Landel RF, Fredericson M. Effects of a single session of posterior-to-anterior spinal mobilization and press-up exercise on pain response and lumbar spine extension in people with nonspecific low back pain. Phys Ther. 2008;88:485-93. 